\title{
Atovaquone-HSA Nano-drugs Enhance the Efficacy of PD-1 Blockade Immunotherapy by Improving Hypoxic Tumor Microenvironment
}

\section{Simeng Wang}

Xi'an Jiaotong University Medical College First Affiliated Hospital

\section{Xinrui Zhou}

Xi'an Jiaotong University Medical College First Affiliated Hospital

\section{Zekun Zeng}

Xi'an Jiaotong University Medical College First Affiliated Hospital

\section{Mengjun Sui}

Xi'an Jiaotong University Medical College First Affiliated Hospital

\section{Lihong Chen}

Xi'an Jiaotong University Medical College First Affiliated Hospital

\section{Chao Feng}

Xi'an Jiaotong University Medical College First Affiliated Hospital

Chen Huang

Xi'an Jiaotong University

\section{Qi Yang}

Xi'an Jiaotong University Medical College First Affiliated Hospital

\section{Meiju Ji}

Xi'an Jiaotong University Medical College First Affiliated Hospital

Peng Hou ( $\sim$ phou@xjtu.edu.cn )

Xi'an Jiaotong University Medical College First Affiliated Hospital

\section{Research Article}

Keywords: Hypoxic tumor microenvironment, Atovaquone, Anti-PD-1 therapy, Nano-drugs, Tumor targeting

Posted Date: May 17th, 2021

DOI: https://doi.org/10.21203/rs.3.rs-502600/v1

License: (c) (i) This work is licensed under a Creative Commons Attribution 4.0 International License.

Read Full License 


\section{Abstract}

Background: Hypoxia is inherent character of most solid malignancies, leading to the failure of chemotherapy, radiotherapy and immunotherapy. Atovaquone, an anti-malaria drug, can alleviate tumor hypoxia by inhibiting mitochondrial complex $\otimes$ activity. The present study exploits atovaquone/albumin nanoparticles to improve bioavailability and tumor targeting of atovaquone, enhancing the efficacy of anti-PD-1 therapy by normalizing tumor hypoxia.

Methods: We prepared atovaquone-loaded human serum albumin (HSA) nanoparticles stabilized by intramolecular disulfide bonds, termed HSA-ATO NPs. The average size and zeta potential of HSA-ATO NPs were measured by particle size analyzer. The morphology of HSA-ATO NPs was characterized by transmission electron microscope (TEM). The bioavailability and safety of HSA-ATO NPs were assessed by animal experiments. Immunofluorescence and ELISA assays were used to evaluate tumor immune microenvironment.

Results: Our data first verified that atovaquone effectively alleviated tumor hypoxia by inhibiting mitochondrial activity both in vitro and in vivo, and successfully encapsulated atovaquone in vesicle with albumin, forming HSA-ATO NPs of approximately $164 \mathrm{~nm}$ in diameter. We then demonstrated that the HSA-ATO NPs possessed excellent bioavailability, tumor targeting and a highly favorable biosafety profile. When combined with anti-PD-1 antibody, we observed that HSA-ATO NPs strongly enhanced the response of mice bearing tumor xenografts to immunotherapy. Mechanistically, HSA-ATO NPs promoted intratumoral $\mathrm{CD}^{+} \mathrm{T}$ cell recruitment by improving tumor hypoxia microenvironment, thereby enhancing the efficacy of anti-PD-1 immunotherapy.

Conclusion: Our data provide strong evidences showing that HSA-ATO NPs can serve as safe and effective nano-drugs to enhance cancer immunotherapy by improving hypoxic tumor microenvironment.

\section{Introduction}

Over the last decades, malignant cancer has posed a serious threat to public health and its incidence continues to be high $[1,2]$. Up to date, immunotherapy has demonstrated to be a reliable therapeutic approach for most cancers [2-5]. One of the major advantages in cancer immunotherapy involves the antibody-mediated blockade of co-inhibitory "checkpoint" molecules, including cytotoxic T lymphocyteassociated protein 4 (CTLA4) and programmed death-1(PD-1) [2, 4]. Blocking PD-1/programmed death ligand 1(PD-L1) signaling has been proved to have durable antitumor immunity and remarkable clinical responses [6, 7]. Varieties of antibodies against the immune checkpoint proteins, PD-1 and PD-L1, have been widely used for cancer therapy [7]. Although some patients responded to immunotherapy, the majority of patients failed to gain ideal benefit, especially the patients with solid tumors $[4,8,9]$.

In contrast to normal tissues, solid tumors have a more complex and harsher microenvironment [10-13]. Hypoxia is a hallmark of solid tumor physiology, as the proliferation of tumor cells results in deprivation of oxygen due to insufficient blood flow from abnormal tumor microvasculature $[12,14,15]$. Tumor 
hypoxia represents a great challenge in cancer therapy $[12,14,16]$. Intratumoral hypoxia increases tumor aggressing, chemo-resistance and radio-resistance $[12,13]$. In recent years, many studies have reached a consensus that hypoxia could limit the efficacy of immunotherapy [17-19]. Meanwhile, there is evidence showing that normalizing tumor hypoxia microenvironment has a robust immune response $[19,20]$. Minimizing mitochondrial oxygen consumption is considered to be one of the most effective approaches to eliminate hypoxia $[20,21]$. Atovaquone, a small molecule drug, was approved by the Food and Drug Administration (FDA) to treat malaria and other diseases [22]. Previous studies indicated that atovaquone could decrease the oxygen consumption of solid tumors by inhibiting the mitochondrial complex $\mathbb{Z}$ activity, eventually alleviating tumor hypoxia [22].

Due to the solubility of atovaquone in water is extremely low, oral preparation remains the most common dosage form for drug delivery $[22,23]$. However, its low bioavailability greatly limits its clinical application. Given that albumin is a biocompatible and safe carrier, and most clinically approved nanodrugs are formulated in albumin [24,25], thus albumin-based delivery system is a good choice to overcome solubility problem of atovaquone. Human serum albumin (HSA) is typically used for drug delivery due to its globular extremely stable structure, which be formed by internal 17 pairs of disulfide bonds [26]. During the process of disulfide bond disconnecting and reconnecting, atovaquone can be enwrapped in albumin, forming a nanoparticle. This approach will improve the bioavailability of the drugs and provide a tumor-targeting effect. Nanoparticles (NPs) are particularly attractive carriers for anticancer drugs because of their efficient cellular internalization and "enhanced permeability and retention (EPR)" properties $[27,28]$. Besides, nano-albumin can be enriched in tumor sites by targeting to glycoprotein 60 (gp60) $[24,25]$.

In this study, we fabricated stable HSA nanoparticles loaded with atovaquone with a redox-responsive character, termed HSA-ATO NPs, and demonstrated that HSA-ATO NPs had excellent tumor-targeting property, and enhanced the efficacy of anti-PD-1 therapy by improving tumor hypoxia.

\section{Materials And Methods}

\section{Synthesis and preparation of HSA-ATO NPs}

To cleave disulfide bonds of HSA to free sulfhydryl groups, HSA was added to deionized water containing $50 \mathrm{mmol} / \mathrm{L} \mathrm{GSH}$ (glutathione) for $4-6 \mathrm{~h}$ at $37^{\circ} \mathrm{C}$. One $\mathrm{mL}$ of DMSO containing $10 \mathrm{mg}$ of atovaquone was then added dropwise into $10 \mathrm{~mL}$ the above solution with slow stirring speed for $15 \mathrm{~min}$. Next, $100 \mu \mathrm{L}$ $\mathrm{H}_{2} \mathrm{O}_{2}$ (30\% aqueous) was continuously added into the suspension to recover intermolecular disulfide bonds. Finally, the suspension was purified by dialysis (MWCO $10 \mathrm{kDa}$ ) followed by a freeze-drying to remove free atovaquone and DMSO.

\section{Physicochemical characteristics of HSA-ATO NPs}

The average size and zeta potential of HSA-ATO NPs was measured by dynamic light scattering (DLS, Zetasizer NanoZS, Malvern, Worcestershire, UK). The morphology of HSA-ATO NPs was characterized by 
transmission electron microscope (TEM) (JEOL JEM-1200EX, Tokyo, Japan). To determine the drug loading and entrapment efficiency (EE), HSA-ATO NPs was first treated with tyrisin $1: 2(\mathrm{v} / \mathrm{v})$ at $37^{\circ} \mathrm{C}$ for 2 $\mathrm{h}$, and then diluted in $2 \mathrm{~mL}$ of DMSO and sonicated for another $30 \mathrm{~min}$ to completely extract atovaquone. The atovaquone amount was determined on a high performance liquid chromatography (HPLC) system. Briefly, HPLC analyses were performed at $40^{\circ} \mathrm{C}$ on a Waters XBridge C18 column $(4.6 \times 150 \mathrm{~mm}, 3.5 \mu \mathrm{m})$ running a $45 \mathrm{~min}, 5-95 \%$ linear gradient of acetonitrile in water containing $0.1 \%$ TFA at a flow rate of 1 $\mathrm{mL} / \mathrm{min}$.

\section{Cell culture}

Murine colon adenocarcinoma cell line MC38 was obtained from the American Type Culture Collection (ATCC; Manassas, VA, USA), and routinely cultured in Dulbecco's Modified Eagle Medium (DMEM) (Gibco, Grand Island, NY) supplemented with $10 \%$ fetal bovine serum (FBS).

In vitro redox-responsive behaviors of HSA-ATO NPs

The ATO release experiment of HSA-ATO NPs was determined by dialysis. Briefly, $1 \mathrm{~mL}$ of purified HSAATO NPs $(0.5 \mathrm{mg} / \mathrm{mL}$ ATO) solution was sealed into a dialysis bag (MWCO $100 \mathrm{kDa})$, and this bag was then immersed into $20 \mathrm{~mL}$ of PBS buffer $(\mathrm{pH} 7.4)$ containing $0.1 \%(\mathrm{v} / \mathrm{v})$ Tween 80 with or without 10 $\mathrm{mmol} / \mathrm{L} \mathrm{GSH}$. To maintain the silk condition, the solution was shaken at $100 \mathrm{rpm}$ at $37^{\circ} \mathrm{C}$. Next, an aliquot of release solution $(200 \mu \mathrm{L})$ at $48 \mathrm{~h}$ was withdrawn for HPLC assay.

\section{Seahorse assay}

Using a Seahorse XFe96 Bioanalyzer (Agilent, Agilent Technologies, USA), MC38 cells (15,000/well) were plated on Seahorse culture plates in Seahorse required medium. Basal oxygen consumption rates (OCR) were taken for $20 \mathrm{~min}$. Cells were then stimulated with $2 \mathrm{mmol} / \mathrm{L}$ oligomycin, $0.5 \mathrm{mmol} / \mathrm{L} \mathrm{FCCP}$ and 100 $\mathrm{mmol} / \mathrm{L}$ rotenone/antimycin A to obtain maximal respiratory and control values.

\section{Evaluation of tumor hypoxia microenvironment}

At 90 min before tumors were surgically excised, pimonidazole hydrochloride (Hypoxyprobe ${ }^{\mathrm{TM}}$ RedAPC Kit, Hypoxyprobe, USA) was intravenously injected into mice at a dose of $50 \mathrm{mg} / \mathrm{kg}$. The paraffin-embedded tissue slides were prepared, and then stained with anti-pimonidazole antibody-APC (Hypoxyprobe) and Hoechst 33342 (ThermoFisher, Beijing, China) after dewaxing and rehydrating. Finally, the sections were imaged with a confocal microscope (Leica, Leica Microsystems).

\section{Animal studies}

The NOD/SCID and BALB/c nude mice (Beijing Vital River Laboratory Animal Technology Co., Ltd. Beijing, China) were used to establish patient-derived xenograft (PDX) model. In brief, tumor tissues were obtained from primary colon cancer tissue after surgical resection, and stored on iced DMEM supplemented with $10 \%$ FBS and antibiotics. Next, these tissues were immediately cut into $1-3 \mathrm{~mm}^{3}$ pieces, and subcutaneously implanted into the flanks of NOD/SCID mice. When tumor volume reached $1-2 \mathrm{~cm}^{3}$, xenograft tumors were harvested and cut into $1-3 \mathrm{~mm}^{3}$ pieces, and subcutaneously implanted 
into the BALB/c nude mice. When xenograft tumors grew to $100 \mathrm{~mm}^{3}$, (1) HSA-ATO NPs, (2) HSA and (3) PBS were intraperitoneally injected for 7 days. Tumor weight and volume were measured before xenograft tumors were harvested for further analysis.

The xenograft tumor model was similarly established as described previously [29]. When the average tumor volume reached $\approx 50 \mathrm{~mm}^{3}$, the mice bearing tumor xenografts were then randomly divided into different groups (five mice per group), and the treatment was begun. HSA (60 mg/kg), HSA-ATO NPs (60 $\mathrm{mg} / \mathrm{kg}$, Atovaquone $\approx 6 \mathrm{mg} / \mathrm{kg}$ ), atovaquone $(60 \mathrm{mg} / \mathrm{kg}$ ) and anti-PD-1 (100 $\mu \mathrm{g}$ each time) were administered at respective dose, with PBS as a negative control. Tumor volume was calculated, and immunohistochemical staining was then performed as described previously [29]. All of the experimental procedures were performed according to Institution Guidelines and approved by Laboratory Animal Center of Xi'an Jiaotong University.

\section{In vivo biodistribution analysis}

At $6 \mathrm{~h}$ after treatment with a single dose of HSA-ATO NPs, the mice were sacrificed and the main organs and tumors were then isolated. Next, about $200 \mathrm{mg}$ tissues were ground and homogenized followed by a $12 \mathrm{~h}$ DMSO extraction, and subjected to HPLC analysis.

\section{Biosafety evaluation of HSA-ATO NPs}

The C57BL/6J xenograft tumor model was used to evaluate the biosafety of HSA-ATO NPs. Tumors and major organs (including heart, liver, spleen, lung and kidney) were collected from mice with different treatments for hematoxylin and eosin (H\&E) assay. All of the images were obtained under an Olympus DP71 microscope. The examinations of liver and kidney function, including the levels of alanine transaminase (ALT), aspartate aminotransferase (AST), serum creatinine (CRE) and blood urea nitrogen (BUN), were performed as described previously [29].

\section{Immunofluorescence staining}

The fluorescently labeled antibodies were used to detect $C D 4^{+} T$ cells and $C D 8^{+} T$ cells in tumor sites. Briefly, tumor tissue were cut into $4 \mu \mathrm{m}$ section and then followed by dewaxing hydration. After blocking with $5 \%$ BSA for 20 min at room temperature, the tissues were incubated the primary anti-CD 4 antibody (Rabbit) and anti-CD8 antibody (Mouse) at $4^{\circ} \mathrm{C}$ overnight. The sections were then washed and incubated Alex 555-conjugated goat anti-mouse secondary antibody and Alex 488-conjugated goat anti-rabbit secondary antibody for marking CD4 + and CD8 + cells, respectively. All antibodies were diluted at $1: 200$, and the nucleus was represented by DAPI. Images were obtained using a confocal microscope (Leica).

\section{Enzyme-linked immunosorbent assay (ELISA)}

The Granzyme and IFN-y content in tumor sites from mice were examined by the Mouse ELISA Kit (Jianglai Bio, Shanghai, China) according to the manufacturer's protocol. The values were normalized by tissue weight. 


\section{Statistical analysis}

All statistical analyses were conducted using the SPSS statistical package (16.0, SPSS Inc. Chicago, IL). Unpaired student's $t$ test was used to compare the means of two groups of data. All values were expressed as the mean \pm standard deviation (SD). All values with $P<0.05$ were considered significantly.

\section{Results}

\section{Atovaquone effectively improves tumor hypoxia by inhibiting mitochondrial activity of tumor cells}

To validate the inhibitory effect of atovaquone on mitochondrial activity of tumor cells, we assessed tumor cells' ability by Seahorse Bioanalyzer by measuring oxidative phosphorylation (OXPHOS), such as oxygen consumption rates (OCR). Before measuring OCR, a cytotoxic experiment was performed to define proper concentrations for subsequent experiments. We found that no more than $10 \mu \mathrm{M}$ atovaquone almost did not affect the proliferation of MC38 cells (Additional file: Figure S1a), while $50 \mu \mathrm{M}$ atovaquone showed pronounced cytotoxicity (Additional file: Figure S1b). Thus, we treated MC38 cells with $1 \mu \mathrm{M}$ atovaquone for $48 \mathrm{~h}$ and performed mitochondrial stress test. As shown in Fig. 1a and b, OXPHOS process of MC38 cells was significantly suppressed by atovaquone compared to the control. To further determine whether atovaquone can improve tumor hypoxia, we first established a MC38 xenograft tumor model. When xenograft tumors reaching $100 \mathrm{~mm}^{3}$, each mouse was administered intragastrically with a single dose of $60 \mathrm{mg} / \mathrm{kg}$ (approximate $1.2 \mathrm{mg}$ each mouse) atovaquone or sterile water once a day for 7 days. With the help of hypoxia-probes, atovaquone treatment clearly decreased overall tumor hypoxia (Fig. 1c). Taken together, our data indicate that atovaquone improves tumor hypoxia by inhibiting mitochondrial activity of tumor cells.

\section{Preparation and characterization of HSA-ATO NPs}

The preparation process of HSA-ATO NPs was shown in Fig. 2a. About $10 \mathrm{mg}$ atovaquone were caught in $100 \mathrm{mg}$ HSA inclusion during the albumin process of denaturation and renaturation, thereby forming nanoparticles. To further investigate the physicochemical properties and loading parameters of HSA-ATO NPs, multiple experiments were shown the characterizations in Table 1. For examples, the zeta potential of HSA-ATO NPs was about $-12.13 \mathrm{mV}$. The drug loading content of formulations was around $10 \%$, with nearly $90 \%$ showing satisfactory entrapment efficiencies. Meanwhile, the morphology of HSA-ATO NPs was represented by TEM (Fig. 2b), and mean size of HSA-ATO NPs was about $164.5 \mathrm{~nm}$ (Fig. 2c). To verify whether atovaquone was successfully loaded, we tested various parameters reflecting atovaquone using UV spectrophotometry and HPLC. As shown in Fig. 2d, a new minor peak appeared at $260 \mathrm{~nm}$ showing that atovaquone was loaded favorably by albumin. As for the depolymerization character of HSA-ATO NPs, we investigated atovaquone releasing response in PBS buffer ( $\mathrm{pH} 7.4)$ with or without 10 $\mathrm{mmol} / \mathrm{L} \mathrm{GSH}$, mimicking the concentration of GSH in tumor cells. After dialysis for $48 \mathrm{~h}$, more than $80 \%$ of loaded atovaquone was able to release in PBS buffer with GSH group, while only $30 \%$ of the loaded 
drug was released in PBS buffer without GSH group (Fig. 2e). Collectively, our data indicate that HSA-ATO NPs are successfully constructed as nanoparticles with satisfactory properties.

Table 1

Physical characterization of HSA-ATO NPs

\begin{tabular}{|ll|}
\hline HSA-ATO NPs & Mean \pm SD $(\mathbf{n}=\mathbf{3})$ \\
\hline Size $(\mathrm{nm})$ & $164.5 \pm 10.3$ \\
\hline PDI & $0.326 \pm 0.02$ \\
\hline Zeta potential $(\mathrm{mV})$ & $-12.13 \pm 1.05$ \\
\hline Drug loading $(\%)$ & $8.70 \pm 1.92$ \\
\hline EE $(\%)$ & $87.2 \pm 2.5$ \\
\hline PDI: Polydispersity index; EE: Encapsulation efficiency \\
\hline
\end{tabular}

\section{HSA-ATO NPs alleviate tumor hypoxia in a PDX model of colon cancer}

To better determine the effect of HSA-ATO NPs on tumor hypoxia in a relatively real tumor microenvironment, we established a PDX mouse model of colon cancer, and administrated these mice with PBS (control), HSA and HSA-ATO NPs (atovaquone at a dosage of $6 \mathrm{mg} / \mathrm{kg}$ ) for 7 days. As expected, HSA-ATO NPs almost did not affect tumor growth (Fig. 3a-c), while obviously alleviated tumor hypoxia compared to the control and HSA treatment (Fig. 3d). Our data indicated that HSA-ATO NPs was able to effectively improve hypoxia environment in solid tumors at a relatively low atovaquone dose, significantly increasing the bioavailability of atovaquone in comparison with previous studies [22].

\section{HSA-ATO NPs maintain a highly biocompatible profile}

Although all raw materials used in this study are FDA-approved, the biosafety of HSA-ATO NPs is still required further investigation. First of all, we examined drug biodistribution in major organs (including heart, liver, spleen, lung and kidneys) and tumor sites. In general, this kind of nanoparticles with a suitable size $(\approx 150 \mathrm{~nm})$ shows a preferable tumor-targeting capacity. As expected, atovaquone was mainly enriched in tumor and liver at $6 \mathrm{~h}$ after HSA-ATO NPs injection (Fig. 4a). Meanwhile, 15 mice were treated with PBS (control), HSA and HSA-ATO NPs for 7 consecutive days. At day 8, major organs and serums were removed and collected to evaluate the biosafety of HSA-ATO NPs. As shown in Fig. 4b and c, HSAATO NPs did not affect the liver and kidney weight of mice compared to the mice administered with HSA or PBS. The results of H\&E staining further demonstrated that HSA-ATO NPs did not cause significant systemic toxicity in vivo compared to the control and HSA treatment (Fig. 4d). Besides, there were no notable changes among these three groups in related safety indicators, including the levels of aspartate aminotransferase (AST), alanine transaminase (ALT), serum creatinine (CRE) and blood urea nitrogen (BUN) (Fig. 4e). Altogether, our data demonstrate that HSA-ATO NPs maintain a highly biocompatible profile. 


\section{HSA-ATO NPs enhance the efficacy of PD-1 blockade immunotherapy}

It is well-known that blocking PD-1/PD-L1 signaling by PD-1 antibody has been widely used for clinical treatment of cancers [30]. To determine whether HSA-ATO NPs can synergize with anti-PD-1 therapy by improving hypoxia environment, we established a homografting colon cancer model by subcutaneously injecting MC38 cells into C57BL6J mice. When the tumors reaching 50-70 $\mathrm{mm}^{3}$, all mice were randomly divided into six groups (five mice per group): 1) PBS (control); 2) anti-PD-1; 3) HSA-ATO NPs + anti-PD-1; 4) HSA-ATO NPs; 5) HSA; 6) atovaquone + anti-PD-1. As shown in Fig. 5a-c, combined treatment of HSAATO NPs and anti-PD-1 synergistically inhibited tumor growth compared to anti-PD-1 or HSA-ATO NPs treatment alone, even better than combined treatment of atovaquone and anti-PD-1. Although traditional intragastric administration of atovaquone also appeared to have a synergistical effect when combining with anti-PD-1, the dose of atovaquone in this manner reached at $60 \mathrm{mg} / \mathrm{kg}$, which is far exceeding the HSA-ATO NPs $(6 \mathrm{mg} / \mathrm{kg})$.

Notably, despite the Ki-67 levels in tumor tissues did not show any significant difference among combined treatment, anti-PD-1 or HSA-ATO NPs treatment alone, the number of TUNEL-positive cells in the tumors with combined treatment of ATO NPs and anti-PD-1 was much higher than that in the tumors with anti-PD-1 or HSA-ATO NPs treatment alone (Fig. 5d). Taken together, our data indicate that HSA-ATO NPs effectively sensitize anti-PD-1 therapy, and further demonstrate that HSA-ATO NPs greatly improve the bioavailability and efficacy of atovaquone.

\section{HSA-ATO NPs improve tumor immune microenvironment (TIME)}

To explore how HSA-ATO NPs improve PD-1 blockade immunotherapy, we next investigated the infiltration of cytotoxic T lymphocyte (CTL) in xenograft tumors. CD8 + cytotoxic T cells, as the most common CTLs, are a key effector in tumor cell eradication. As shown in Fig. 6a and b, compared to the control, the number of $C D 8+T$ cell infiltration was visibly increased when the mice were treated with either atovaquone, HSA-ATO NPs or anti-PD-1. However, combined treatment of HSA-ATO NPs and antiPD-1 resulted in the largest accumulation of CTL in tumor tissues compared to other treatments. Meanwhile, we also found that CD8 + T cell infiltration in the tumors with combined treatment of HSAATO NPs and anti-PD-1 was the most obvious compared to other treatments (Fig. 6a and b). Besides, the granzyme and IFN- $\gamma$, commonly associated with the immune cell activities, were clearly elevated by combined treatment of HSA-ATO NPs and anti-PD-1 compared to other treatments (Fig. $6 \mathrm{c}$ and d). These observations indicate that HSA-ATO NPs enhance the efficacy of anti-PD-1 therapy by effectively improving the TIME.

\section{Discussion}


In this study, we link hypoxic tumor microenvironment to the resistance of immunotherapy, further highlighting the importance of tumor hypoxic microenvironment in cancer therapy. Our results reveal that hypoxia is an important barrier for $\mathrm{T}$ cell

infiltration, thereby limiting the efficacy of immunotherapy. Improving tumor microenvironment by inhibition of tumor cell oxidative metabolism will significantly increase sensitivity to immunotherapy, unleashing antitumor immune response to promote cancer regression [20, 31, 32]. This was strongly supported by our data that both quantity and activity of the immune cells were increased after improving tumor microenvironment [33,34]. Taken together, the situation of tumor environment directly determines the efficacy of cancer immunotherapy. Thus, tumor microenvironment has to be considered for cancer therapy, especially hypoxia environment $[35,36]$.

In general, two strategies "direct delivery of oxygen to tumor" and "reduce tumor oxygen consumption" are often designed to improve tumor hypoxia. However, the latter method is more advantageous for achieving with the drugs. There is evidence showing that normalizing tumor hypoxia by existing drugs is experimentally feasible for clinical treatment of cancers [22]. In this study, our data strongly indicated that atovaquone, an FDA-approved drug for malaria treatment, significantly alleviated the harsh hypoxic tumor microenvironment. Although other drugs can also achieve similar effects by inhibiting tumor oxygen consumption, atovaquone exhibits the most powerful effect on lowering tumor oxygen consumption. More importantly, it does not exert toxic effects for major organs at an adequate dose to improve hypoxia [20]. However, the extremely low water-solubility of atovaquone leads to its poor bioavailability, thereby greatly limiting its clinical utility. It is very necessary to improve its bioavailability in order to meet clinical needs. Thus, designing a proper delivery system will be an effective strategy to address the above mentioned dilemma.

Albumin has good biocompatibility and biodegradability, thus serving as a versatile carrier for drug delivery [37]. One of the best known examples is albumin-bound paclitaxel (nab-PTX), which has been used in clinic for years [38]. Paclitaxel is enwrapped into albumin to form stable nanoparticles with a suitable size in order to enhance the bioavailability of PTX [38]. Considering that atovaquone is as hydrophobic as PTX, this kind of nanoparticle will provide an effective strategy to solve delivery problem of atovaquone [39]. Not surprisingly, our data showed that the albumin delivery system was able to load atovaquone, which formed nanoparticles named HSA-ATO NPs. Compared to traditional oral drug administration, the dose of HSA-ATO NPs to normalize tumor hypoxia was drastically reduced by as much as 10 -fold. Under the premise of the same curative effect, this kind of nano-drugs greatly reduces the dose of atovaquone for clinical treatment. Moreover, due to the EPR effect and specific albumin receptors in malignant tumors, HSA-ATO NPs possessed the excellent targeting efficiency to tumor [24, $27,40]$. This also verified that albumin delivery system increased the bioavailability of the loaded drugs and maintained a highly biocompatible profile.

It is the fact that clinical tumors are highly heterogeneous in terms of tumor microenvironment containing endothelial cells, pericytes, immune cells, fibroblasts and extracellular matrix (ECM) [41-43]. The 
xenograft tumor model derived from a single cell line can not fully reflect complete tumor microenvironment, while PDX model will effectively mimic the native tumor microenvironment $[44,45]$. In this study, our data demonstrated that the HSA-ATO NPs effectively alleviated tumor hypoxia in PDX mouse model, indicating that this kind of nano-drug has a great potential for the treatment of cancer patients. Importantly, all raw materials used in this study are FDA-approved, thus HSA-ATO NPs are all considered as safe and reliable in clinic. Based on this system, a number of improvements also could be made. One of them is that make the checkpoint antibodies directly conjugate to albumin by unique chemical modifications. As a consequence, this will form a more complex nano-drugs to solve a variety of problems, such as the drugs with low bioavailability or poor tumor targeting. In the near future, we may carry out other combined treatment regimens by this safe and targeting delivery system. Thus, we are going to persist exploiting this system to investigate more issues.

\section{Conclusion}

In summary, we develop a novel nano-drug by using HSA to load atovaquone, and demonstrate that this nano-drug enhances the efficacy of PD-1 blockade immunotherapy by improving tumor hypoxia microenvironment, thereby providing a potential strategy for cancer therapy.

\section{Abbreviations}

HSA: Human serum albumin; PD-1: programmed death-1; PD-L1: programmed death ligand 1; FDA: Food and Drug Administration; TEM: Transmission electron microscope; NPs: Nanoparticles; ELISA: Enzymelinked immunosorbent assay; CD8: Cluster of differentiation 8; CD4: Cluster of differentiation 4; CTLA4: Cytotoxic T lymphocyte-associated protein 4; EPR: Enhanced permeability and retention; gp60:

Glycoprotein 60; DMSO: Dimethylsulfoxide; EE: Entrapment efficiency; HPLC: High performance liquid chromatography; DMEM: Dulbecco's Modified Eagle Medium; FBS: Fetal bovine serum; GSH: glutathione; OCR: oxygen consumption rates; PDX: Patient-derived xenograft; H\&E: Hematoxylin and eosin; AST: Aspartate aminotransferase; ALT: Alanine transaminase; BUN: Blood urea nitrogen; CRE: Creatinine; OXPHOS: Oxidative phosphorylation; IFN- $\gamma$ : Interferon- $\gamma$; TIME: Tumor immune microenvironment; $\mathrm{CTL}$ : cytotoxic T lymphocyte; nab-PTX: Albumin-bound paclitaxel; PTX: paclitaxel; ECM: Extracellular matrix

\section{Declarations}

\section{Ethics approval and consent to participate}

All animal experimental procedures were approved by the Animal Ethics Committee of Xi'an Jiaotong University.

\section{Consent for publication}

All authors read and approved the final manuscript. 


\section{Availability of data and materials}

The datasets generated and analyzed during the current study are available from the corresponding author on reasonable request.

\section{Competing interests}

P.H., S.W., and M.J. are co-authors of a patent been filed with Chinese patent application no. 2021110095184.4. The authors declare that they have no other competing interests.

\section{Funding}

This work was supported by the National Natural Science Foundation of China (No. 82072949 to P.H.; No. 81972593 to M.J.), Innovation Talent Promotion Plan in Shaanxi Province (No. 2018TD-006 to P.H.), the Science and Technology Project of Shaanxi Province (No. 2019SF-015 to P.H.) and the Shaanxi Key Research and Development Program (No. 2021 SF-109 to Q.Y.)

\section{Authors' contributions}

P.H. and M.J. conceived and designed the experiments. S.W., X.Z., Z.Z., M.S., C.F., L.C., and Q.Y. performed the experiments and analyzed the data. M.J. and P.H. contributed reagents and materials. S.W. and P.H. wrote the manuscript. All authors read and approved the final manuscript.

\section{Acknowledgements}

Not applicable.

\section{References}

1. Siegel RL, Miller KD, Jemal A. Cancer statistics, 2020. Ca-Cancer J Clin. 2020;70:7-30.

2. Anderson KG, Stromnes IM, Greenberg PD. Obstacles Posed by the Tumor Microenvironment to T cell Activity: A Case for Synergistic Therapies. Cancer Cell. 2017;31:311-25.

3. Yang EY, Shah K. Nanobodies: Next Generation of Cancer Diagnostics and Therapeutics. Front Oncol. 2020;10.

4. de Miguel M, Calvo E. Clinical Challenges of Immune Checkpoint Inhibitors. Cancer Cell. 2020;38:32633.

5. Blatner NR, Bonertz A, Beckhove P, Cheon EC, Krantz SB, Strouch M, et al. In colorectal cancer mast cells contribute to systemic regulatory T-cell dysfunction. P Natl Acad Sci USA. 2010;107:6430-5. 
6. Togashi Y, Shitara K, Nishikawa H. Regulatory T cells in cancer immunosuppression - implications for anticancer therapy. Nat Rev Clin Oncol. 2019;16:356-71.

7. Constantinidou A, Alifieris C, Trafalis DT. Targeting Programmed Cell Death-1 (PD-1) and Ligand (PDL1): A new era in cancer active immunotherapy. Pharmacol Ther. 2019;194:84-106.

8. Duan JC, Cui LG, Zhao XC, Bai H, Cai SL, Wang GQ, et al. Use of Immunotherapy With Programmed Cell Death 1 vs Programmed Cell Death Ligand 1 Inhibitors in Patients With Cancer A Systematic Review and Meta-analysis. JAMA Oncol. 2020;6:375-84.

9. Zarour HM. Reversing T-cell Dysfunction and Exhaustion in Cancer. Clin Cancer Res. 2016 15;22:1856-64 .

10. Bahrami A, Khazaei M, Hassanian SM, ShahidSales S, Joudi-Mashhad M, Maftouh M, et al. Targeting the tumor microenvironment as a potential therapeutic approach in colorectal cancer: Rational and progress. J Cell Physiol. 2018;233:2928-36.

11. Spano D, Zollo M. Tumor microenvironment: a main actor in the metastasis process. Clin Exp Metastas. 2012;29:381-95.

12. Patel A, Sant S. Hypoxic tumor microenvironment: Opportunities to develop targeted therapies. Biotechnol Adv. 2016;34:803-12.

13. Li JY, Chen YP, Li YQ, Liu N, Ma J. Chemotherapeutic and targeted agents can modulate the tumor microenvironment and increase the efficacy of immune checkpoint blockades. Mol Cancer. 2021;20:27.

14. Kim Y, Lin Q, Glazer PM, Yun Z. Hypoxic Tumor Microenvironment and Cancer Cell Differentiation. Curr Mol Med. 2009;9:425-34.

15. Dhani N, Fyles A, Hedley D, Milosevic M. The Clinical Significance of Hypoxia in Human Cancers. Semin Nucl Med. 2015;45:110-21.

16. Daniel SK, Sullivan KM, Labadie KP, Pillarisetty VG. Hypoxia as a barrier to immunotherapy in pancreatic adenocarcinoma. Clin Transl Med. 2019;8.

17. Vaupel P, Multhoff G. Hypoxia-/HIF-1 alpha-Driven Factors of the Tumor Microenvironment Impeding Antitumor Immune Responses and Promoting Malignant Progression. Adv Exp Med Biol. 2018;1072:171-5.

18. Labiano S, Palazon A, Melero I. Immune Response Regulation in the Tumor Microenvironment by Hypoxia. Semin Oncol. 2015;42:378-86.

19. Munn DH, Bronte V. Immune suppressive mechanisms in the tumor microenvironment. Curr Opin Immunol. 2016;39:1-6.

20. Scharping NE, Menk AV, Whetstone RD, Zeng X, Delgoffe GM. Efficacy of PD-1 Blockade Is Potentiated by Metformin-Induced Reduction of Tumor Hypoxia. Cancer Immunol Res. 2017;5:9-16.

21. Secomb TW, Hsu R, Ong ET, Gross JF, Dewhirst MW. Analysis of the Effects of Oxygen-Supply and Demand on Hypoxic Fraction in Tumors. Acta Oncol. 1995;34:313-6. 
22. Ashton TM, Fokas E, Kunz-Schughart LA, Folkes LK, Anbalagan S, Huether M, et al. The anti-malarial atovaquone increases radiosensitivity by alleviating tumour hypoxia. Nat Commun. 2016;7.

23. Ke F, Yu JQ, Chen W, Si XM, Li XH, Yang F, et al. The anti-malarial atovaquone selectively increases chemosensitivity in retinoblastoma via mitochondrial dysfunction-dependent oxidative damage and Akt/AMPK/mTOR inhibition. Biochem Biophs Res Commun. 2018;504:374-9.

24. Sleep D. Albumin and its application in drug delivery. Expert Opin Drug Deliv. 2015;12:793-812.

25. Merlot AM, Kalinowski DS, Richardson DR. Unraveling the mysteries of serum albumin-more than just a serum protein. Front Physiol. 2014;5.

26. Jiang LQ, Xu YS, Liu Q, Tang Y, Ge L, Zheng CL, et al. A nontoxic disulfide bond reducing method for lipophilic drug-loaded albumin nanoparticle preparation: Formation dynamics, influencing factors and formation mechanisms investigation. Int J Pharm. 2013;443:80-6.

27. Maeda $\mathrm{H}, \mathrm{Wu} J$, Sawa T, Matsumura Y, Hori K. Tumor vascular permeability and the EPR effect in macromolecular therapeutics: a review. J Control Release. 2000;65:271-84.

28. Fang J, Islam W, Maeda $\mathrm{H}$. Exploiting the dynamics of the EPR effect and strategies to improve the therapeutic effects of nanomedicines by using EPR effect enhancers. Adv Drug Deliv Rev. 2020;157:142-60.

29. He WX, Wang SM, Yan J, Qu YP, Jin L, Sui F, et al. Self-Assembly of Therapeutic Peptide into StimuliResponsive Clustered Nanohybrids for Cancer-Targeted Therapy. Adv Funct Mater. 2019;29.

30. Jiang $X$, Wang J, Deng X, Xiong F, Ge J, Xiang B, et al. Role of the tumor microenvironment in PDL1/PD-1-mediated tumor immune escape. Mol Cancer. 2019;18:10.

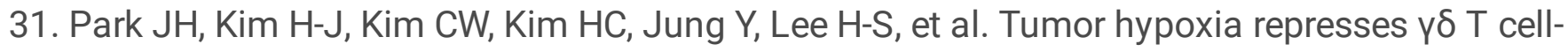
mediated antitumor immunity against brain tumors. Nat Immunol. 2021;22:336-46.

32. Palazón A, Martínez-Forero I, Teijeira A, Morales-Kastresana A, Alfaro C, Sanmamed MF, et al. The HIF-1 a hypoxia response in tumor-infiltrating $T$ lymphocytes induces functional CD137 (4-1BB) for immunotherapy. Cancer Discov. 2012;2:608-23.

33. Jayaprakash P, Ai MD, Liu A, Budhani P, Bartkowiak T, Sheng J, et al. Targeted hypoxia reduction restores $\mathrm{T}$ cell infiltration and sensitizes prostate cancer to immunotherapy. J Clin Invest. 2018;128:5137-49.

34. Scarfo I, Maus MV. Current approaches to increase CAR T cell potency in solid tumors: targeting the tumor microenvironment. J Immunother Cancer. 2017;5:28.

35. Noman MZ, Chouaib S. Targeting hypoxia at the forefront of anticancer immune responses. Oncoimmunology. 2015 7;3:e954463.

36. Scharping NE, Rivadeneira DB, Menk AV, Vignali PDA, Ford BR, Rittenhouse NL, et al. Mitochondrial stress induced by continuous stimulation under hypoxia rapidly drives $T$ cell exhaustion. Nat Immunol. 2021;22:205-15.

37. Chen J, Ma XY, Dong QC, Song DH, Hargrove D, Vora SR, et al. Self-healing of thermally-induced, biocompatible and biodegradable protein hydrogel. Rsc Adv. 2016;6:56183-92. 
38. da Costa R, Passos GF, Quintao NLM, Fernandes ES, Maia JRLCB, Campos MM, et al. Taxaneinduced neurotoxicity: Pathophysiology and therapeutic perspectives. Brit J Pharmacol. 2020;177:3127-46.

39. Ruan CH, Liu LS, Lu YF, Zhang Y, He X, Chen XL, et al. Substance P-modified human serum albumin nanoparticles loaded with paclitaxel for targeted therapy of glioma. Acta Pharm Sin B. 2018;8:85-96.

40. Zhao C, Song X, Liu Y, Fu Y, Ye L, Wang N, et al. Synthesis of graphene quantum dots and their applications in drug delivery. J Nanobiotechnology. 2020;18:142.

41. Prasetyanti PR, Medema JP. Intra-tumor heterogeneity from a cancer stem cell perspective. Mol Cancer. 2017;16:41.

42. Fane $M$, Weeraratna AT. How the ageing microenvironment influences tumour progression. Nat Rev Cancer. 2020;20:89-106.

43. Kaczanowska S, Beury DW, Gopalan V, Tycko AK, Qin H, Clements ME, et al. Genetically engineered myeloid cells rebalance the core immune suppression program in metastasis. Cell. 2021;184:203352.

44. Saito Y, Shultz LD, Ishikawa F. Understanding Normal and Malignant Human Hematopoiesis Using Next-Generation Humanized Mice. Trends Immunol. 2020;41:706-20.

45. Bagherifar R, Kiaie SH, Hatami Z, Ahmadi A, Sadeghnejad A, Baradaran B, et al. Nanoparticlemediated synergistic chemoimmunotherapy for tailoring cancer therapy: recent advances and perspectives. J Nanobiotechnology. 2021;19:110.

\section{Figures}



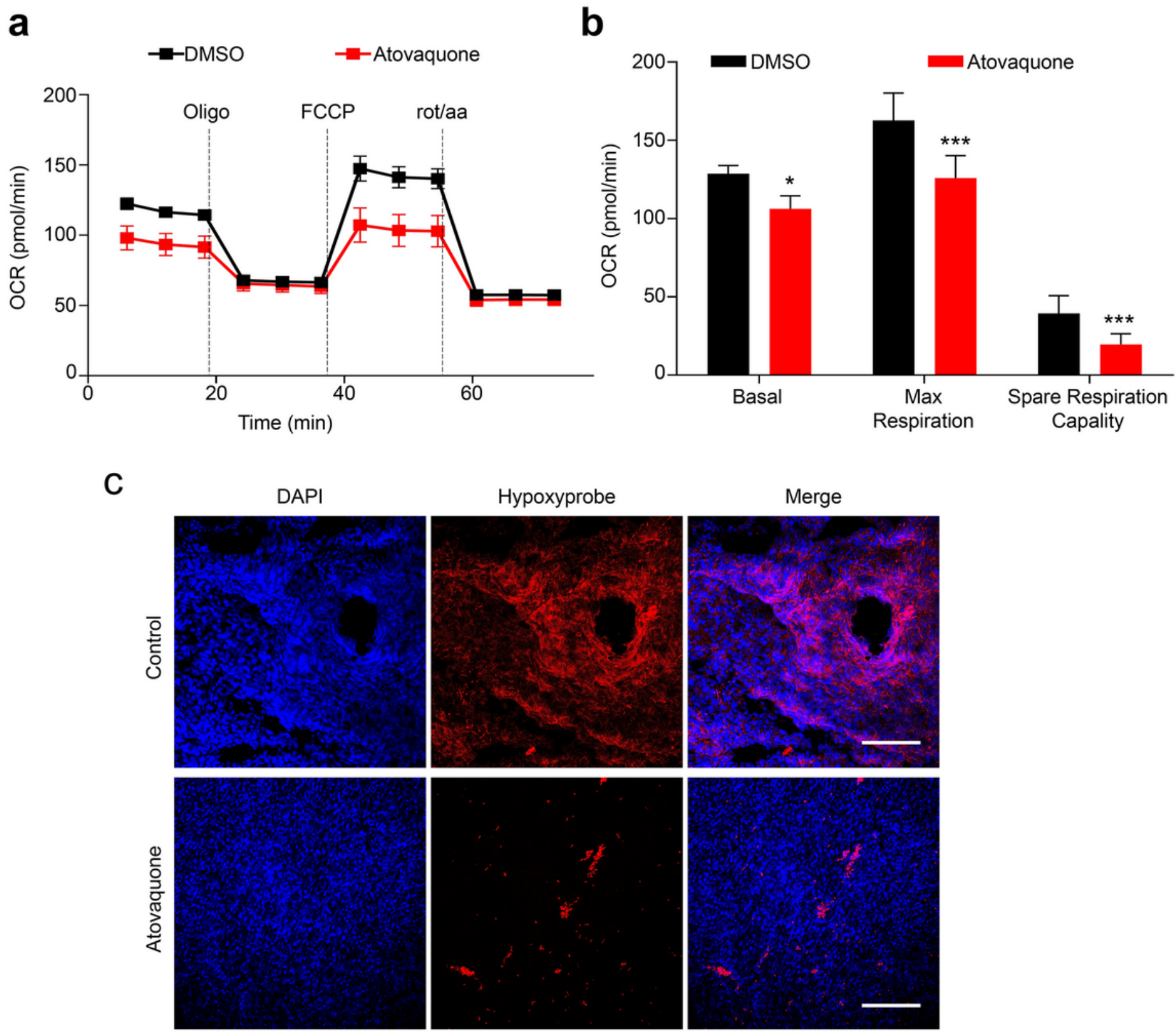

\section{Figure 1}

Improvement of tumor hypoxia by atovaquone via inhibiting mitochondrial complex activity of tumor cells. a, b OCR trace of MC38 cells (15,000 cells/well) interrogated for mitochondrial activity in the Seahorse instrument. c APC-Hypoxia probe staining of tumor sections from mice bearing MC38 tumors receiving PBS or atovaquone treatment for 7 days. Scale bar: $200 \mu \mathrm{m}$. Data are presented as mean \pm SD. $\star, P<0.05 ; * \star, P<0.01 ; * \star *, P<0.001$. 


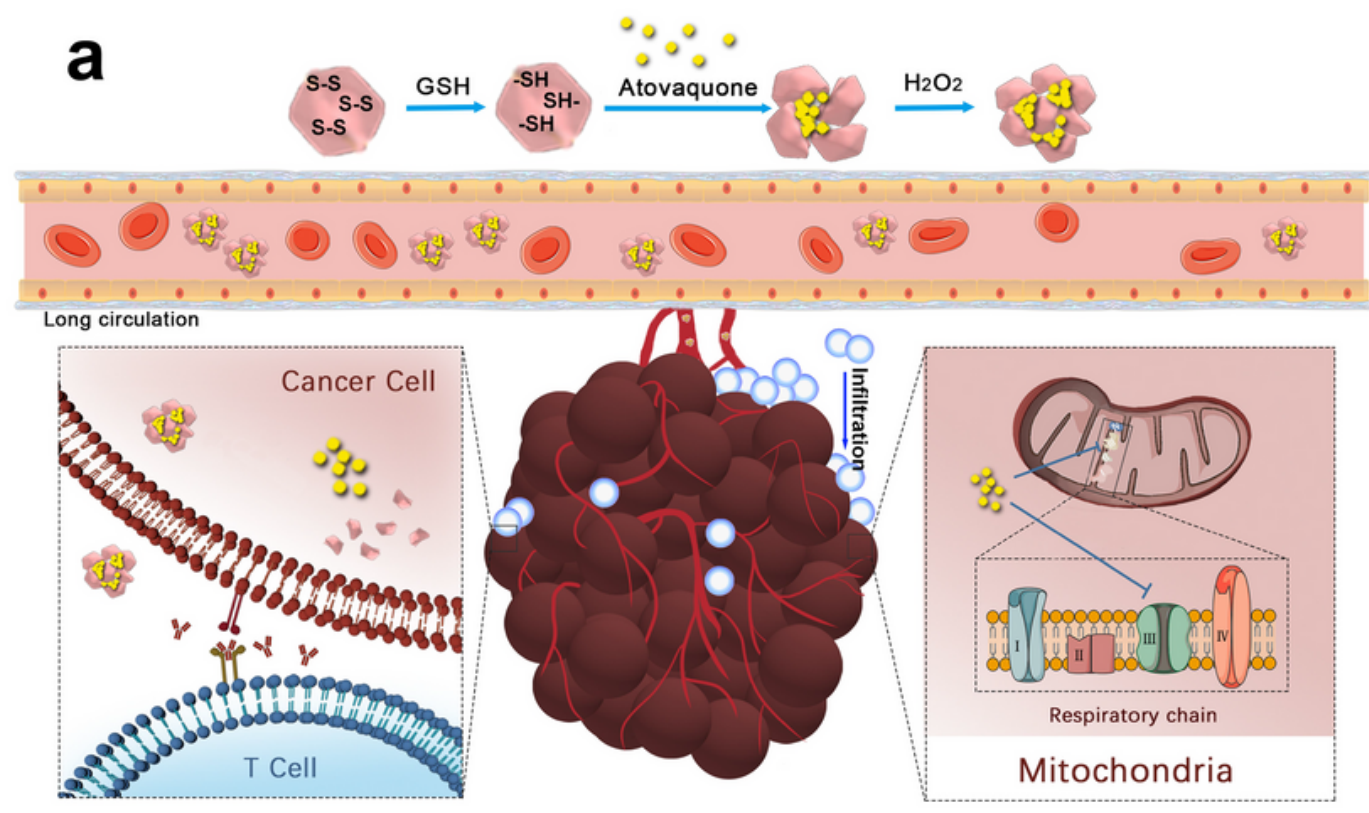

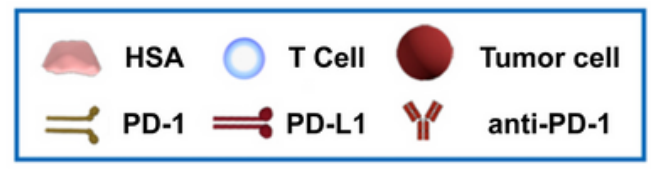

b

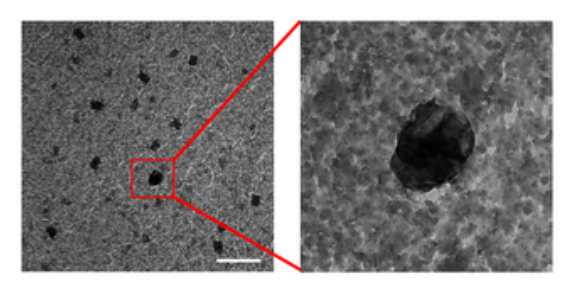

d

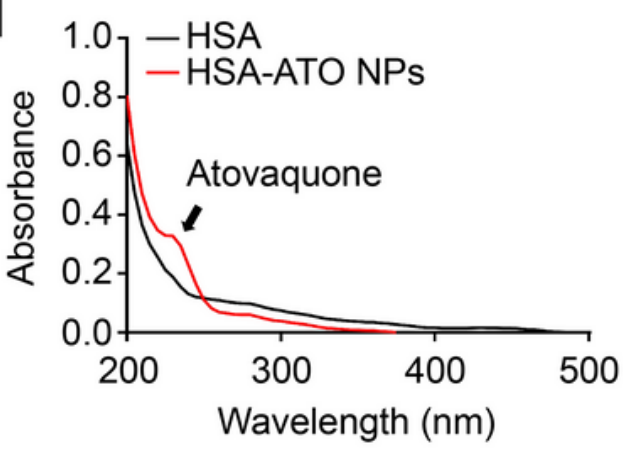

Atovaquone improves tumor hypoxia by inhibiting respiratory chain complex III

C

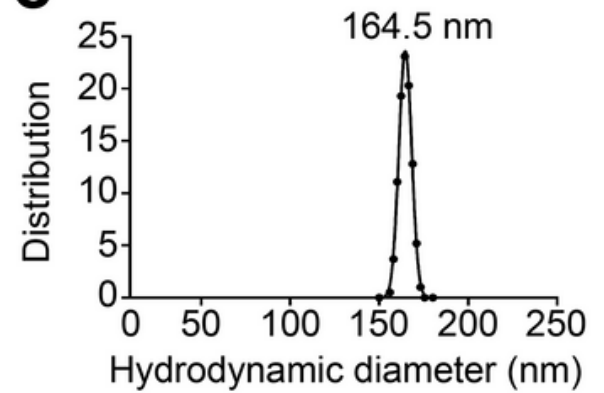

e

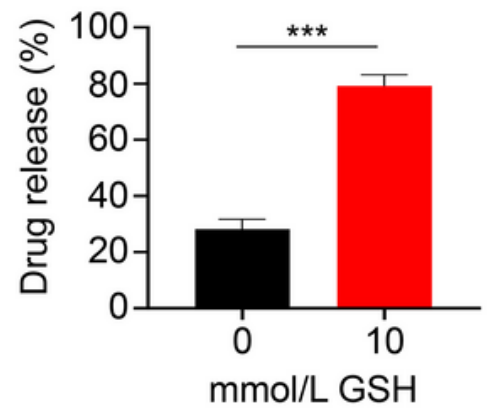

\section{Figure 2}

Preparation and characterization of HSA-ATO NPs. a Schematic depiction for synthesis and function of HSA-ATO NPs. b TEM image of HSA-ATO NPs, presenting an amorphous form. Scale bar: $1 \mu \mathrm{m}$. c Hydrodynamic distributions of HSA-ATO NPs measured by dynamic light scattering. $d$ The full wavelength detection of HSA-ATO NPs and HSA were detected by UV spectrophotometry. e In vitro drug 
release profiles of HSA-ATO NPs in different mediums. Data are presented as mean \pm SD. ${ }^{*}, \mathrm{P}<0.05 ; * \star, P$ $<0.01 ; * \star \star, P<0.001$.
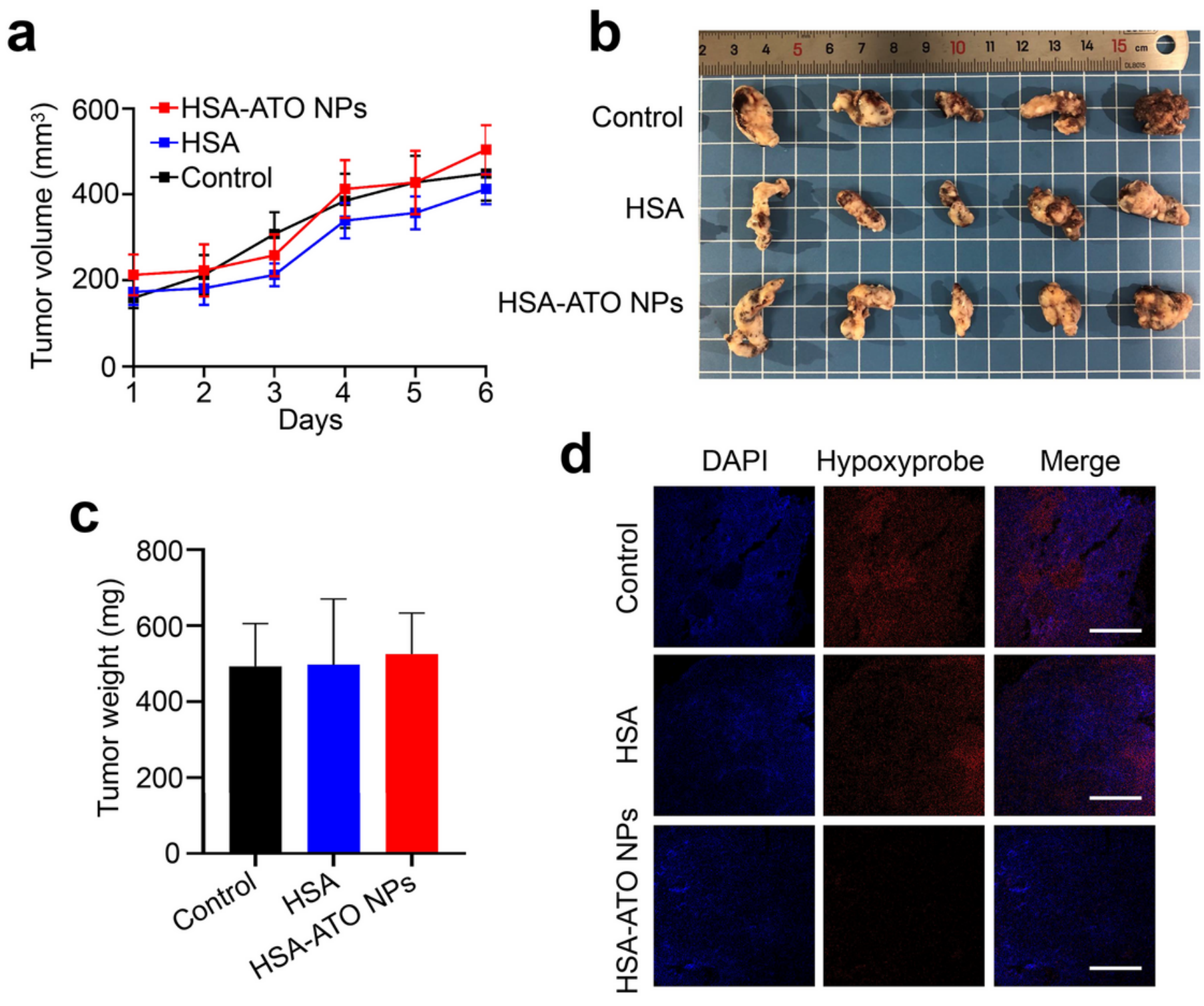

Figure 3

Improvement of tumor hypoxia by HSA-ATO NPs in PDX mouse model. a Tumor growth curve of volume according time during the administration ( $n=5)$. Photographs (b) and weight (c) of tumors collected from mice after 7-day administration $(n=5)$. d APC-Hypoxia probe staining of tumor sections from mice bearing PDX tumors receiving PBS, HSA or HSA-ATO NPs treatment for 7 days. Scale bar: $500 \mu \mathrm{m}$. Data are presented as mean $\pm \mathrm{SD}$. *, $\mathrm{P}<0.05 ; * \star, \mathrm{P}<0.01 ; * \star \star, \mathrm{P}<0.001$. 
a

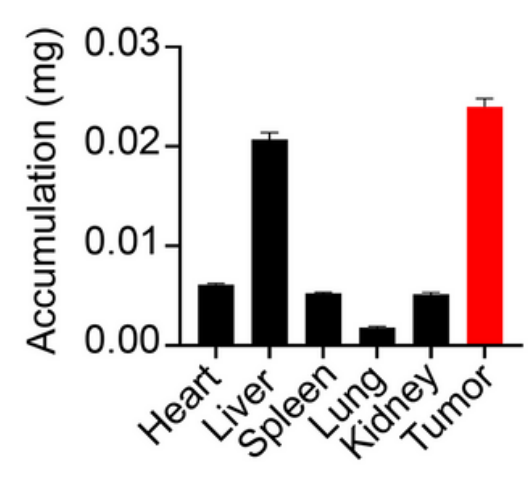

d

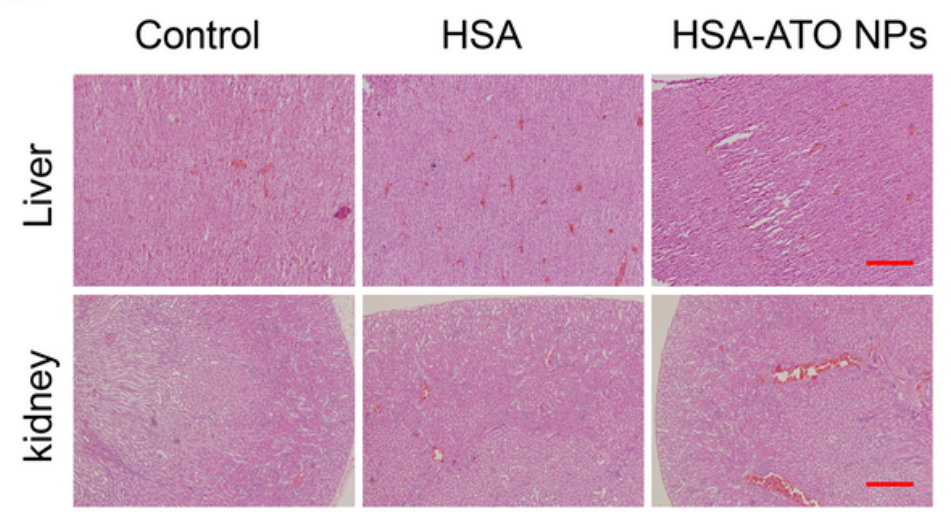

C
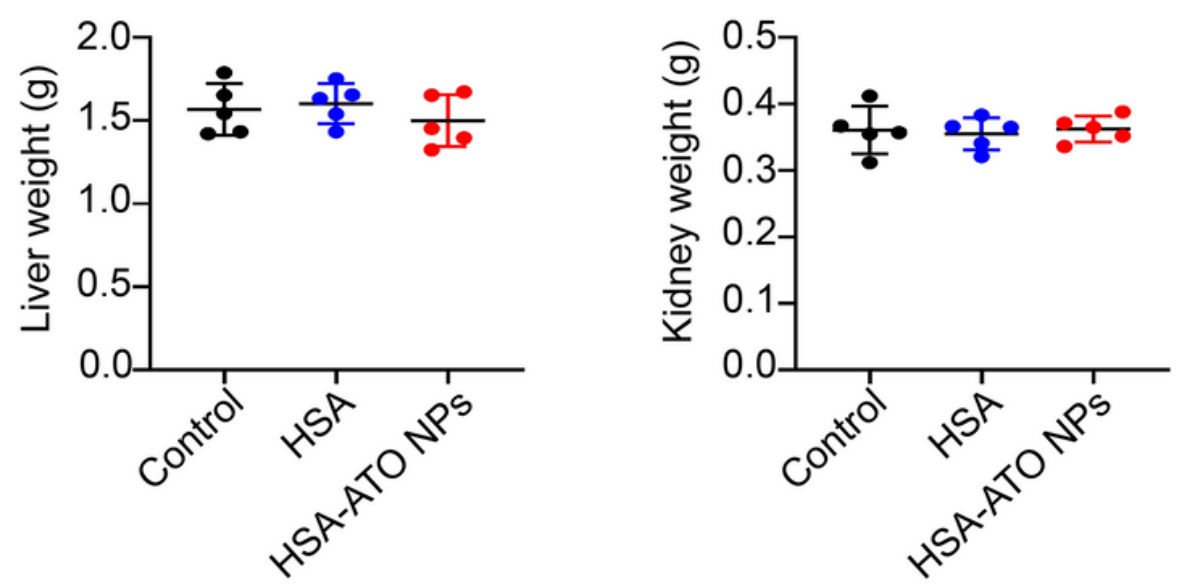

e
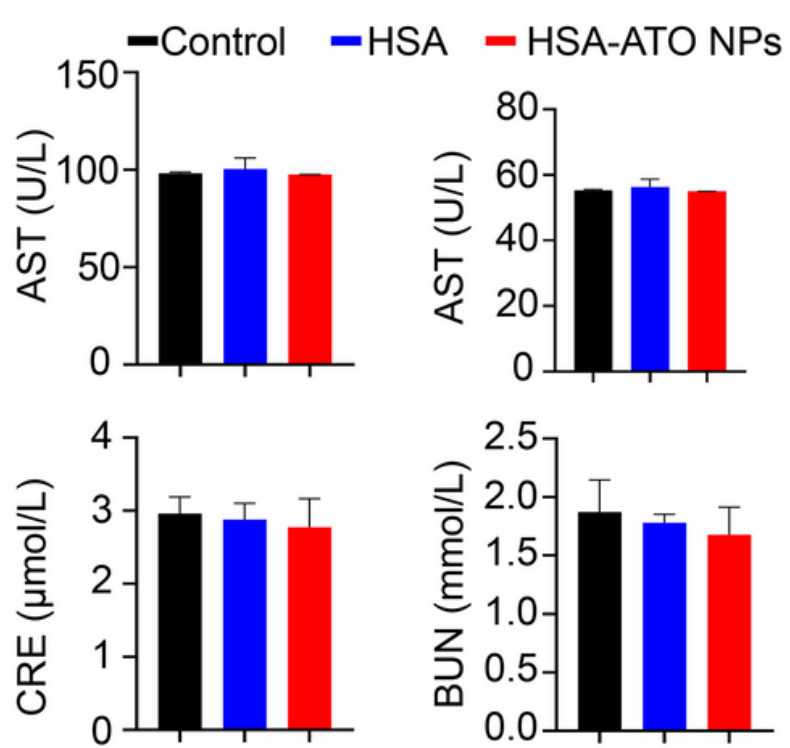

Figure 4

Biosafety evaluation of HSA-ATO NPs. a The relative accumulation quality of atovaquone in major organs and tumor sits was analyzed by HPLC. The weight of liver (b) and kidney (c) of the indicated mice with 7-day administration ( $n=5)$. $d$ The representative images of H\&E-stained liver and kidney sections in mice with the indicated treatments. Scale bar: $500 \mu \mathrm{m}$. e The levels of aspartate aminotransferase (AST), alanine transaminase (ALT), blood urea nitrogen (BUN) and serum creatinine (CRE) were performed by ELISA assay. Data are presented as mean \pm SD. 

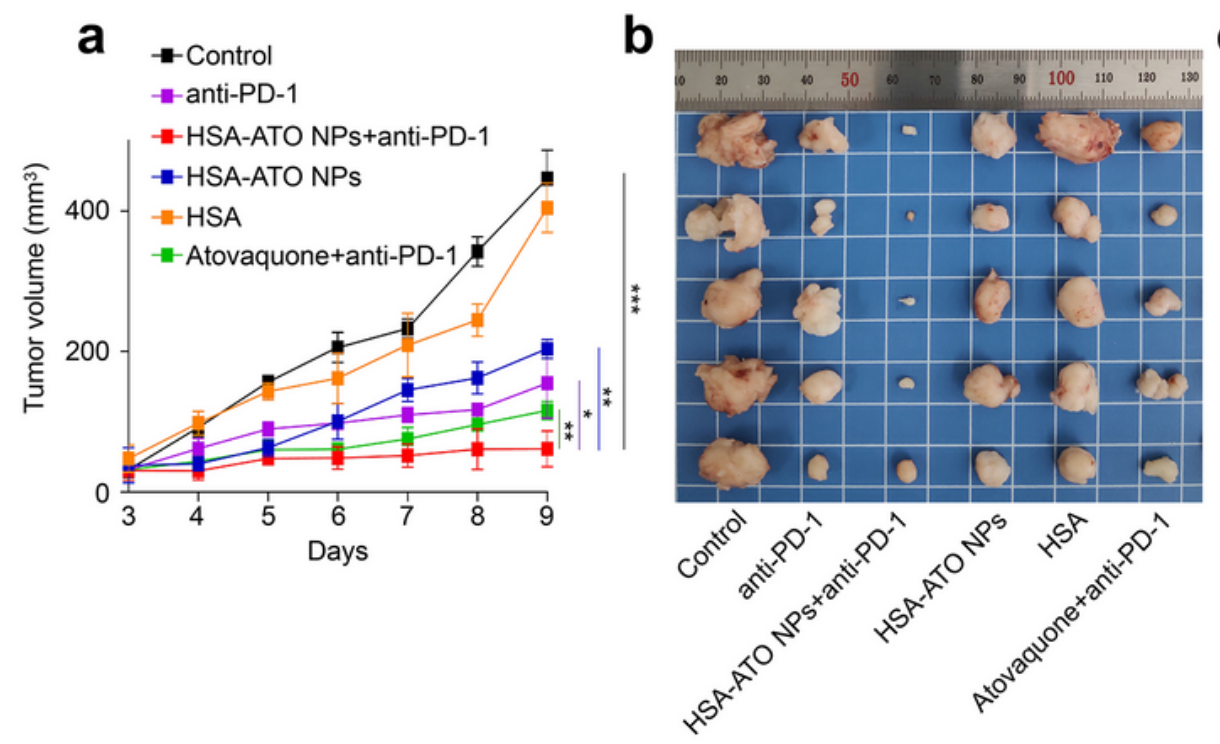

C

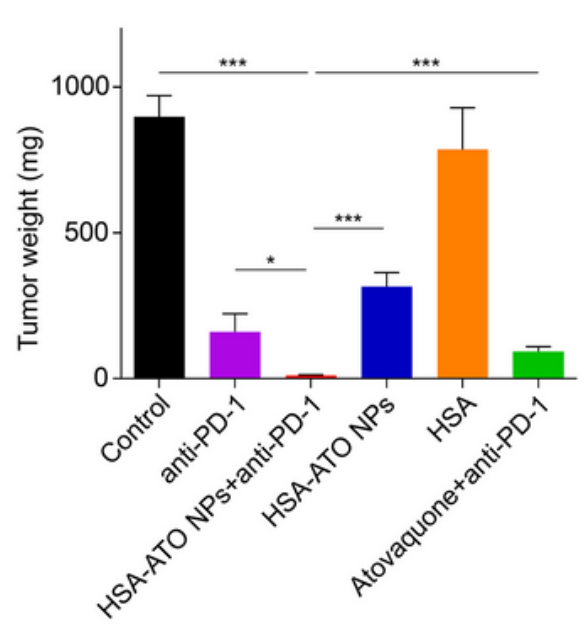

d

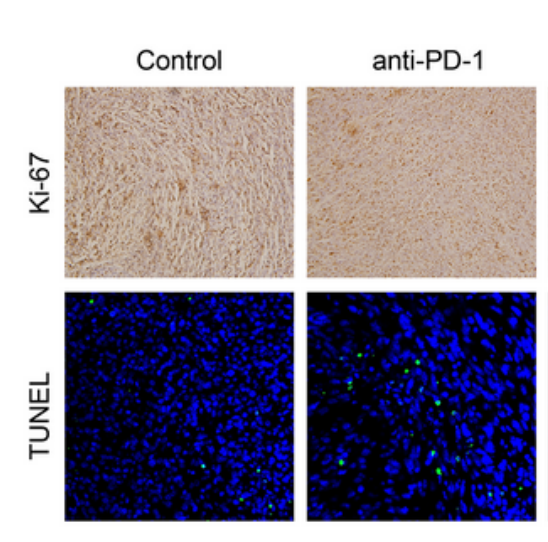

HSA-ATO NPS + anti-PD-1
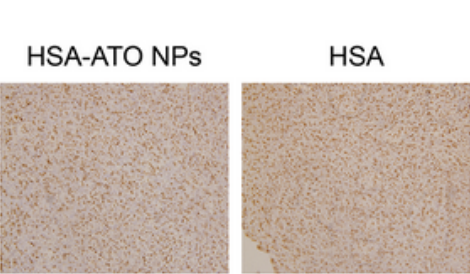

Atovaquone
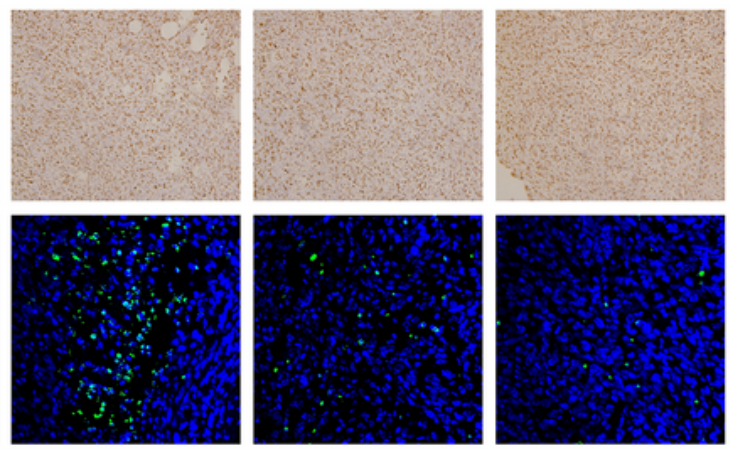

+ anti-PD-1
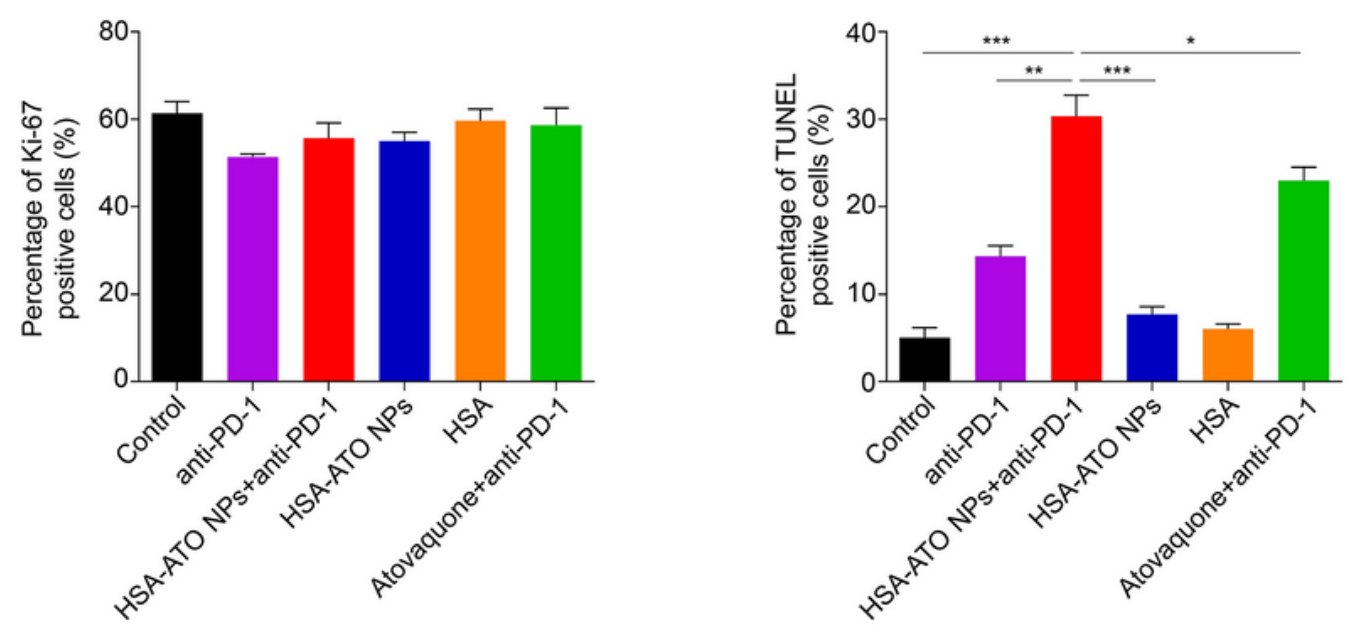

\section{Figure 5}

HSA-ATO NPs synergize with anti-PD-L1 therapy. a Tumor growth curve of volume according time during the administration ( $n=5)$. Photographs (b) and weight (c) of tumors collected from mice after 7-day administration $(n=5)$. $d$ The percentage of Ki-67 and TUNEL positive cells in xenograft tumors was measured by optical or fluorescence microscopes. Data are presented as mean $\pm \mathrm{SD}$. ${ }^{*}, \mathrm{P}<0.05$; $* *, \mathrm{P}<$ $0.01 ; * \star *, P<0.001$. 

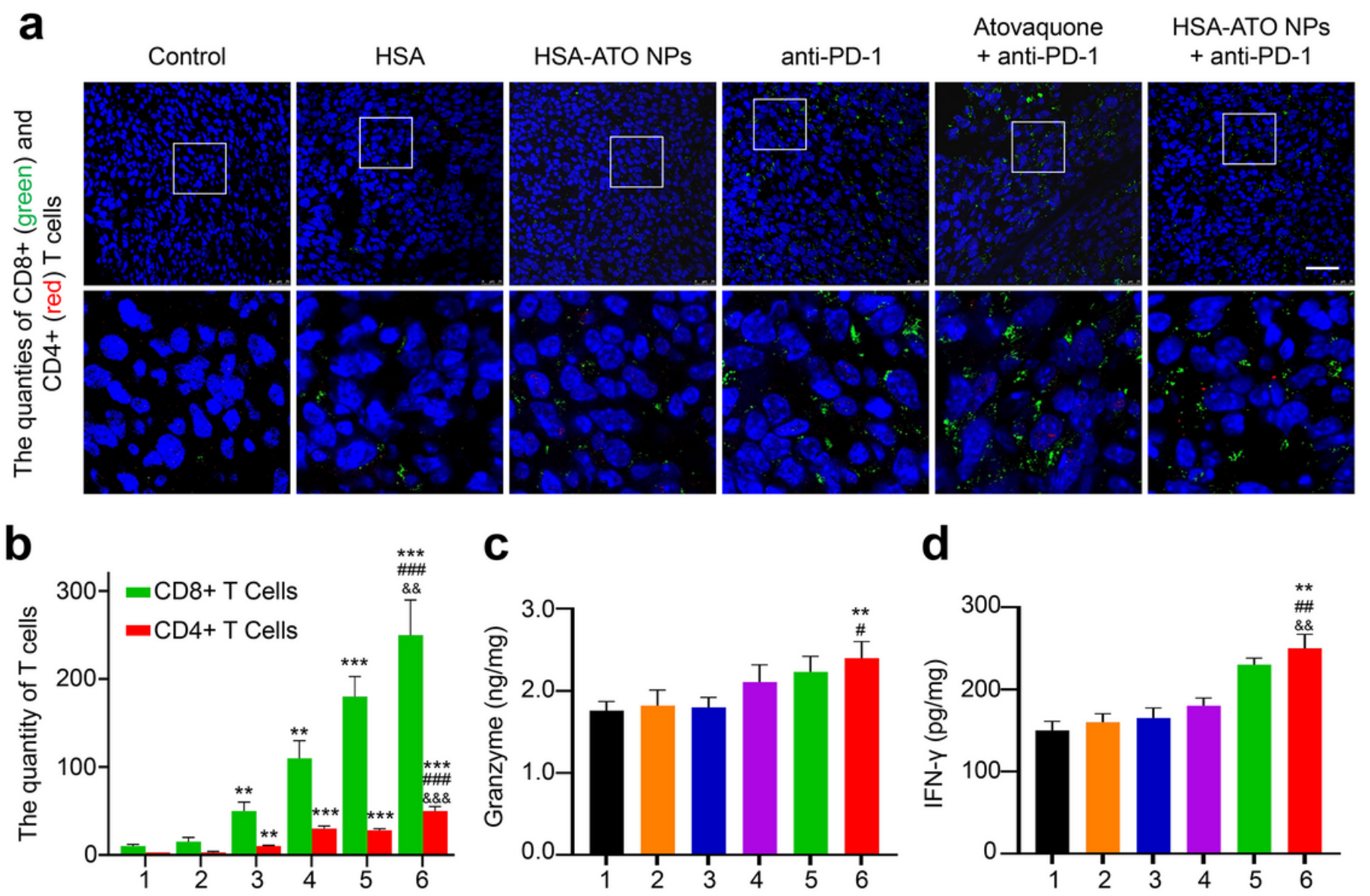
1. Control
2. HSA
3. HSA-ATO NPS
4. anti-PD-1
5. Atovaquone + anti-PD-1
6. HSA-ATO NPs + anti-PD-1

\section{Figure 6}

Improvement of tumor immune microenvironment (TIME) by HSA-ATO NPs. a Immunofluorescence images of CD4+/CD8+ cells in tumor sections from mice with the indicated treatments. Scale bar: $50 \mu \mathrm{m}$. The quantity of immune cells (b) and the content of Granzyme (c) and IFN- $y$ (d) in xenograft tumors from mice with the indicated treatments were analyzed by immunofluorescence and ELISA assays. Data are presented as mean $\pm S D$. **, $\mathrm{P}<0.01$; ***, $\mathrm{P}<0.001$ for comparison with the control; $\#, \mathrm{P}<0.05$; \#\#, $\mathrm{P}<$ 0.01 ; \#\#\#, $\mathrm{P}<0.001$ for comparison with HSA-ATO NPs; \&\&, $\mathrm{P}<0.01 ; \& \& \&, \mathrm{P}<0.001$ for comparison with anti-PD-1 therapy.

\section{Supplementary Files}

This is a list of supplementary files associated with this preprint. Click to download.

- Graphicalabstract.jpg

- Additionalfile.docx 